\title{
Discrimination of two auditory durations by pigeons ${ }^{1}$
}

JULAINE KINCHLA ${ }^{2}$

MCMASTER UNIVERSITY

\begin{abstract}
Discrimination between two auditory durations was investigated in pigeons with a procedure that provided a direct analogue to the conventional "yes-no" signal detection task with humans. In Experiment 1 the development and maintenance of the discrimination using highly confusable durations was examined. Experiment 2 investigated sequential effects in stationary performance. The effect of altering probability of reinforcement and extinction was also examined. The birds developed and maintained a partial discrimination. As separation between the two durations was reduced, discriminability was systematically decreased. Reduction of the probability of reinforcement produced changes similar to those exhibited by humans when pay-off functions are altered. The only sequential effect of any size was a tendency to repeat responses. Individual differences were considerable.
\end{abstract}

The purpose of the present investigation was to develop a discrete-trial procedure for examining duration discrimination in animals, a procedure that would provide data amenable to analysis in terms of a decision-theoretic model of the type exemplified by the psychophysical theory of signal detection (this theory is developed in detail in Green and Swets, 1966). The experimental procedure presented here represents an alternative to other methods that have been used to study duration discrimination (or the related phenomenon of temporal discrimination) in animals, such as a DRL. schedule or schedules employing DRL as one component.

The method for studying discrimination behavior that has been developed and has seen extensive application in psychological experiments with humans has several distinguishing features, i.e., it is a discrete-trial, noncorrection, choice procedure. It allows the presentation of two or more stimulus values, one of which occurs on every trial. The $E$, not the $S$, controls the stimulus, enabling its presentation on a predetermined schedule with known statistical properties. The $S$ must make a choice response after each presentation and is not allowed to correct.

In the typical signal-detection experiment, $S$ is required to identify which of two possible stimulus events, denoted $S_{1}$ and $S_{2}$, occurs in each of a series of trials. If the $S$ 's report that an $S_{1}$ or $S_{2}$ stimulus was presented is denoted by $A_{1}$ or $A_{2}$ respectively, his performance can then be summarized by two proportions: the proportion of $A_{1}$ responses on $S_{1}$ trials, denoted by $\dot{P}\left(A_{1} \mid S_{1}\right)$, and the proportion of $A_{1}$ responses on $S_{2}$ trials, denoted by $\mathbf{P}\left(A_{1} \mid S_{2}\right)$. These two proportions are normally interpreted as estimates of two corresponding theoretical probabilities: $P\left(A_{1} \mid S_{1}\right)$ and $P\left(A_{1} \mid S_{2}\right)$. These probabilities are often called "hits" and "false alarms," respectively (e.g., Green \& Swets, 1966).

The central assumption of signal detection theory is that each presentation of a particular stimulus value $\left(S_{\mathfrak{i}}\right)$ evokes a value of a hypothetical sensory variable that can be represented as a Gaussian random variable. The distribution of sensory values evoked by an $S_{i}$ stimulus event can be characterized by their expected value, denoted by $\mu_{\mathbf{i}}$, and standard deviation, denoted $\sigma_{i}$. If the distributions of sensory states evoked by two stimulus values are similar, the $S$ will have difficulty discriminating the stimuli. It is assumed that he determines his response on the basis of a decision criterion, $c$, reporting that stimulus value with the higher expected sensory value only if the sensory value on that trial is greater than c. Thus there are two separate aspects of the discrimination process: the similarity of the distribution of sensory values evoked by $S_{1}$ and $S_{2}$, and the response criterion adopted by the $S$. The similarity of the two sensory distributions is usually characterized by two measures, $d^{\prime}$ and $k$, where

$$
\mathrm{d}^{\prime}=\frac{\mu_{1}-\mu_{2}}{\sigma_{2}}
$$

and

$$
\mathrm{k}=\frac{\sigma_{2}}{\sigma_{1}}
$$

It is assumed that $\mu_{1}$ is greater than $\mu_{2}$.

Data in these experiments are often reported in the form of ROC curves. To obtain these, the two conditional probabilities $P\left(A_{1} \mid S_{1}\right)$ and $P\left(A_{1} \mid S_{2}\right)$ are plotted in a two-dimensional graph. Note that a point falling in the extreme upper left of the graph indicates perfect discrimination, while points falling on the positive diagonal reflect no discrimination, and intermediate points indicate intermediate levels of partial discrimination. The curve that results as the S's criterion is systematically varied is called an ROC curve, the receiver-operating characteristic, which, according to detection theory, should be symmetric about the negative diagonal when $k=1$ (the "equal variance" assumption), and pass through the points 0,0 and 1,1 . (For a more detailed discussion of the theory of signal detection, see Green and Swets, 1966.)

Several recent studies have demonstrated the fruitfulness of a decision-theoretic approach in discrimination studies with animals. Experiments have been reported in which light intensity (Nevin, 1964; Morrison, 1967; Keuchler, 1968), tone (Hack, 1963), and gustatory substances (Morrison \& Norrison, 1966) have been used as stimuli. Taken together, the results from these experiments were consistent with signal detection theory as a model of discrimination in animals for a variety of stimuli.

The first investigation to apply a decision-theoretic approach to duration discrimination was a study by Creelman (1962) with human Os. Creelman's model was based on a counting mechanism and was used to account for performance in a two-interval forced-choice procedure in which sounds of brief duration (around $1 \mathrm{sec}$ ) were presented. It provided a good fit to the data over a wide range of conditions.

The only attempts to analyze duration discrimination in animals in a form amenable to analysis by Creelman's model (or the signal detection model to which it is related) is that represented in this paper and in another paper by Stubbs (1968). Stubbs used a discrete trial procedure in which a pigeon was presented with 1 of 10 possible durations on each trial and required to make a choice response, i.e., respond to one key color when a short duration tone occurred, and another key color given a long duration tone. Response frequencies were observed to vary in a fashion consistent with the pay-off structure. This would seem to provide evidence that duration discrimination in animals does behave in a fashion similar to 
that predicted by a decision-theory model. However, Stubbs employed more than two stimuli in his discrimination task, which distinguishes it from the more conventional two-stimulus detection problem. The experiments we shall now consider did employ just two stimuli and were designed to provide a direct analogue to the conventional detection task.

In these experiments, pigeons were required to discriminate between two durations of a clearly audible tone stimulus on each of a series of trials. Each trial began with the illumination of a "set-up" key which the pigeon must peck to initiate the presentation of the tone stimulus. The tone remained on with equal probability for either $T_{1}$ or $T_{2}$, following which two other keys, one on each side of the set-up key, were illuminated. In order to obtain food reinforcements, the pigeon had to peck one of these keys on $T_{1}$ trials and the other on $T_{2}$ trials. We shall refer to the response that is reinforced on $T_{1}$ trials as an $R_{1}$ response, and that on $T_{2}$ trials as an $R_{2}$ response. The animal's performance on this task will be summarized by the proportion of $R_{2}$ responses on $T_{1}$ trials and the proportion of $R_{2}$ responses on $T_{2}$ trials, denoted by $\hat{p}_{1}$ and $\hat{p}_{2}$, respectively. (These properties will be interpreted as estimates of corresponding theoretical probabilities denoted by $p_{1}$ and $p_{2}$ ). Thus $\hat{\mathrm{p}}_{2}$ and $\hat{\mathrm{p}}_{1}$ correspond to the type of "hit" and "false alarm" measures derived in signal detection analysis. Two other estimates shall also be used to characterize performance, $\dot{\mathbf{p}}_{\mathrm{c}}$ and $\hat{\mathbf{p}}_{\mathrm{L}}$, where $\hat{\mathrm{p}}_{\mathrm{c}}=\mathrm{P}($ correct $)=(1-\gamma) \mathrm{P}\left(\mathrm{R}_{1} \mid \mathrm{T}_{1}\right)+$ $\gamma P\left(R_{2} \mid T_{2}\right)$ and $\hat{p}_{L}=P\left(R_{2}\right)=$ $(1-\gamma) \mathrm{P}\left(\mathrm{R}_{2} \mid \mathrm{T}_{1}\right)^{\circ}+\gamma \mathrm{P}\left(\mathrm{R}_{2} \mid \mathrm{T}_{2}\right)$. The parameter $\gamma$ denotes the fixed probability of a $\mathrm{T}_{2}$ stimulus on each trial.

The experimental work consisted of two main experiments. Experiment 1 was concerned primarily with the problem of shaping the discrimination, particularly when the two temporal durations were so similar that the discrimination was less than perfect. Values of $T_{1}$ and $T_{2}$ were determined that would produce a terminal level of performance where the animal was correct on about $65 \%$ of the trials. Experiment 2 was designed to evaluate the stability of the pigeons' performance during extended training at this level of partial discrimination and, second, to evaluate the effects of altering the probability of reinforcement following a correct response. The latter manipulation could be considered as being analogous to altering the pay-off function and thereby altering the $O$ 's criterion in signal detection experiments.

\section{EXPERIMENT 1}

This experiment involved the gradual key was transilluminated with a white light

shaping of seven pigeons to perform the type of temporal discrimination just described.

\section{Apparatus}

The animals were tested in a standard, two-key testing chamber (Lehigh Valley Electronics, Model 1519) modified by the addition of a third key, center-mounted 3.5 in. above the food hopper. Each key could be illuminated from behind by a white bulb. The sequence of stimulus events in the test chambers was controlled from a room next to that containing the test chambers. White noise and a $1,000-\mathrm{Hz}$ tone were presented in the experimental chamber by a speaker located on the lower left corner of the chamber wall that contained the keys and food hopper. The intensity of the tone and noise stimuli was $80 \pm 10 \mathrm{~dB}$. Accuracy of the temporal durations used in the study were limited by the relay control equipment but were accurate to $\pm .5 \%$ (i.e., to $\pm 25 \mathrm{msec}$ of the final $T_{1}$ and $T_{2}$ values used in Experiment 1).

\section{Subjects}

The Ss were seven male White Carneaux pigeons, approximately 5 years of age and experimentally naive. ${ }^{3}$

\section{Procedure}

The shaping process whereby the animals were trained to operate the set-up key to produce the tone stimulus and then to peck one of the two side keys, consisted of five steps.

Step 1, terminal link of the chain. Magazine training was followed by first shaping a key approach to a lighted key, then a key peck to the lighted key. On each trial, either the right or left key could be transilluminated with a white light according to a random schedule in which each key had an a priori probability of .5 of being selected. A peck to the lighted key darkened the key and produced food for 4 sec. $A$ peck to an unlighted key had no effect. The intertrial interval (ITI) was 5 sec.

Step 2, Link 2 of the chain. After termination of the ITI, the trial began with a $1-\sec$ tone followed by either right or left key light. A single peck to the lighted key darkened it and produced reinforcement. A peck to an unlighted key, including key pecks while the tone was on, had no effect. Reinforcement was decreased to 3-sec access to grain and the ITI was increased to $15 \mathrm{sec}$ (in Experiment 2 it was increased to an interval that varied randomly in $1 / 2-\mathrm{sec}$ intervals from 10 to $20 \mathrm{sec}$ ). A response during the ITI delayed the start of the next trial for $15 \mathrm{sec}$.

Step 3, Link 1 of the chain. The center at the beginning of the trial. A single peck was required to turn the light off and produce Link 2, the tone, for $1 \mathrm{sec}$, at the termination of which either right or left key light came on. The same key peck contingencies as before were in effect in Links 2 and 3 . Pecks to the unlighted side keys in Link 1 had no effect.

Step 4, differentiation $T_{1}$ and $T_{2}$. The time during which the tone was on in the second link was differentiated into two durations, a short duration, $T_{1}$, equal to $1 \mathrm{sec}$, and a long duration, $T_{2}$, which was gradually increased to $5 \mathrm{sec}$ over the session. Presentation of $T_{1}$ and $T_{2}$ was according to a random schedule in which each had an a priori probability of occurrence of .5 .

During this phase of the experiment, $T_{1}$ was always followed by the left key light, $T_{2}$ was followed by the right key light. (In Experiment 2, the above contingency was in effect for a random half of the Ss; for the other half it was reversed.) Pecks to the appropriate key following a $T_{1}$ and $T_{2}$ were designated $R_{1}$ and $R_{2}$, respectively. Only an $R_{1}$ following a $T_{1}$, or an $R_{2}$ following a $T_{2}$, were reinforced. An $R_{1}$ given a $T_{2}$ or an $R_{2}$ given a $T_{1}$ had no effect.

Step 5, brightness fading. At the termination of the tone, i.e.,$T_{1}$ and $T_{2}$, both right and left key lights were transilluminated. However, brightness on the incorrect key was initially very dim. It was gradually increased to full brightness over five sessions. This technique was adapted following results obtained in the studies by Terrace (1966) on the formation of errorless discrimination.

Incorrect responses, i.e., $R_{2} T_{1}$ or $R_{1} T_{2}$, now had the effect of terminating the trial and producing the ITI. Correct responses were reinforced as before.

After this step, key light brightness no longer functioned as a cue for the correct response, and shaping was completed. The only stimulus correlated with a reinforced outcome was the auditory duration. A choice response was now required in the terminal link.

Following this shaping procedure, the animals were presented with the following sequence of events during each discrimination trial: (1) The trial began with the center of the three response keys being lighted and the other two dark; (2) a single peck on the center key turned it off and turned on a $1,000 \mathrm{~Hz}$ tone that stayed on for either $T_{1}$ or $T_{2}$ sec; (3) when the tone went off, both of the two side response keys were lighted simultaneously; (4) a single peck on either key turned the key lights off; (5) if the animal had pecked the $R_{i}$ key and the tone duration was $T_{i}$ $(i=1,2)$ it received a food reinforcement followed by an intertrial interval (ITI), 
Table 1

Procedure for Experiment 1

\begin{tabular}{|c|c|c|c|c|}
\hline \multirow[b]{2}{*}{ Procedure } & \multicolumn{2}{|c|}{ Group 1} & \multicolumn{2}{|c|}{ Group 2} \\
\hline & Sessions & $\begin{array}{c}\text { Total } \\
\text { Number } \\
\text { of Sessions }\end{array}$ & Sessions & $\begin{array}{c}\text { Total } \\
\text { Number } \\
\text { of Sessions }\end{array}$ \\
\hline $\begin{array}{l}\text { Shaping } \\
T_{1}=2 \mathrm{Sec} \\
T_{\text {Transition }} \text { to } \mathrm{T}_{1}=3 \mathrm{Sec} \\
\mathrm{T}_{1}=3 \mathrm{Sec} \\
\text { Fading } \\
\mathrm{T}_{1}=3 \mathrm{Sec} \\
T_{r a n s i t i o n} \text { and Fading } \\
\mathrm{T}_{1}=4 \mathrm{Sec}\end{array}$ & $\begin{array}{r}1-15 \\
16-30 \\
31-33 \\
34-53 \\
54-56 \\
57-66 \\
67-69 \\
70-79 \\
\end{array}$ & $\begin{array}{r}15 \\
15 \\
3 \\
20 \\
3 \\
10 \\
3 \\
10\end{array}$ & $\begin{array}{r}1-15 \\
16-30 \\
31-33 \\
34-38\end{array}$ & $\begin{array}{r}15 \\
15 \\
3 \\
5\end{array}$ \\
\hline
\end{tabular}

otherwise only the ITI occurred.

The $R_{1}$ and $R_{2}$ response keys were always the left and right keys, respectively, in this experiment (although this was varied in Experiment 2). Reinforcement was $3-\mathrm{sec}$ access to grain with the food hopper light on during that time. White noise was on continuously except during the tone period. The ITI was $20 \mathrm{sec}$ during which the chamber was illuminated by a "houselight." If the animal pecked the key during the ITI, it delayed the onset of the next trial by $20 \mathrm{sec}$.

The sequence of $T$ values was determined in blocks of 20 trials to approximate an independent-trials Bernoulli process with $T_{1}$ and $T_{2}$ occurring with equal probability on each trial (both $T$ values occurred equally often in each block of 20 trials but in a randomly determined sequence).

Experimental sessions were given daily, with each session lasting until 90 reinforcements had been obtained. Animals were kept at $80 \%$ normal (ad lib feeding) body weight. If an animal was underweight prior to an experimental session, he was fed upon return to his cage.

Following the shaping process, the animals were given several sessions in which they were required to discriminate a $T_{1}$-equal-2-sec and $T_{2}$-equal-5-sec pair of tone durations. They were then required to make a more difficult discrimination by successively increasing the value of $T_{1}$ from 2 to $4 \mathrm{sec}$ in gradual stages while $T_{2}$ was fixed at $5 \mathrm{sec}$. The number of sessions at each particular pair of $T$ values is summarized in Table 1 . Note that one group of animals, Group 1, was run for 79 sessions, while another group, Group 2, was run for only 51 . The basic difference between these two groups was the extended training of Group 1 with $T_{1}$ equal to $3 \mathrm{sec}$ ( 30 sessions) before moving to the $T_{1}$-equal-to-4-sec condition, as compared to the few sessions (5) with $T_{1}$ equal to $3 \mathrm{sec}$ for Group 2.

The periods of training identified as "fading" in Table 1 involved "brightness fading," that is, lighting only the correct response key, or "temporal fading," that is, producing gradual increments in $T_{1}$. These fading techniques were used to facilitate adjustment to the shifts in $T$ values and were administered in random fashion to the birds in each group. Since the two types of fading procedure produced essentially equivalent behavios, they will not be dealt with further.

\section{Results}

The observed values of $\hat{\mathrm{p}}_{1}$ and $\hat{\mathrm{p}}_{2}$ for each animal at each value of $T_{1}(2,3$, and $4 \mathrm{sec}$ ) are presented graphically in Fig. 1. Figure $1 \mathrm{~A}$ presents these pairs of proportions for the $T_{1}$-equal-2-sec condition, with each animal's performance defining a point $\left(\hat{p}_{2}, \hat{p}_{1}\right)$ in a two-dimensional coordinate system. Similar graphs of the data for the $T_{1}$-equal-3- and 4-sec conditions are presented in Figs. 1B and 1C, respectively. Each point represents the pooled data from five sessions at each $T_{1}$ value, which corresponds to more than 450 trials for each data point. Data for the last five sessions are shown except for the $T_{1}$-equal-3-sec condition, where data from the first five sessions of each group are shown. It is clear from simple visual inspection that the performance points were successively displaced away from the upper left corner of the graph toward the line $\dot{p}_{1}$ equal $\dot{p}_{2}$ as $T_{1}$ was made more similar to $T_{2}$, i.e., discrimination became poorer. This successive drop in proportion of correct responses was consistent for all seven animals and is thus statistically significant, $\mathrm{p}<.01$.

When $T_{1}$ was increased to its final value of $4 \mathrm{sec}$ there was a difference of only $1 \mathrm{sec}$ between $T_{1}$ and $T_{2}, A$ group difference appears in which Group 1 appeared to be discriminating better than Group 2. The mean $\mathrm{pC}_{C}$ for the second block of five sessions was .644 for Group 1 and .562 for Group 2.

\section{Discussion}

The results in Figs. 1A, 1B, and 1C suggest that the pigeons behaved in a fashion similar to humans in analogous psychophysical tasks. The shift in
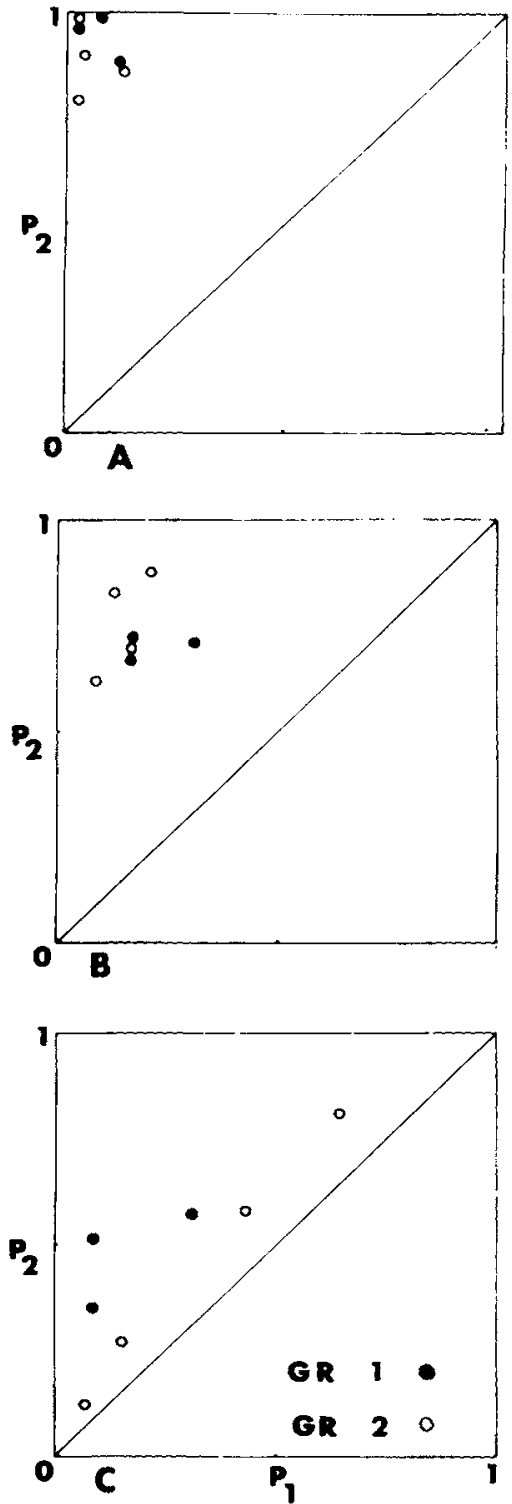

Fig. 1. Estimates of $p_{1}$ and $p_{2}$, based on five sessions, with $T_{2}$ equal to $5 \mathrm{sec}$ and $T_{1}$ equal to: A, $2 \mathrm{sec}$; $B, 3 \mathrm{sec}$; and $C, 4 \mathrm{sec}$.

performance points toward the positive diagonal in Fig. 1 is consistent with an experimental manipulation decreasing the stimulus difference (making discrimination more difficult) in a conventional signal detection task. Furthermore, the substantial individual differences that existed among the birds within a group, make the use of group data highly questionable.

It is of special interest to note that the birds were able to maintain a partial discrimination over an extended series of trials instead of fixating on one response and accepting a simple random ratio reinforcement schedule of .5. For example, 
the overall mean $p_{C}$ at $T_{1}$ equal to $4 \mathrm{sec}$ and $T_{2}$ equal to $5 \mathrm{sec}$ was .599. Some of the birds were performing only slightly better than chance, yet they generally appeared to maintain a consistent degree of partial discrimination.

The effect of differential amount of training on Groups 1 and 2 at $T_{1}$ equal $4 \mathrm{sec}$ suggest the dependence of discrimination on past history. In human signal detection, the theoretical measure $\mathrm{d}^{\prime}$ is considered to be a function of the physical stimulus and the "sensitivity" of the particular $O$. The sensitivity is assumed to be a relatively stable feature of the perceptual process, as opposed to the response bias or criterion $\mathrm{c}$, which is a relatively arbitrary and easily modified feature. The data obtained here, showing better discrimination for Group 1 birds, suggest that a sensitivity measure such as $\mathrm{d}^{\prime}$ may not be a stable feature of the animal's behavior. Relatively long periods of acquisition or training may be required before it becomes stable.

\section{EXPERIMENT 2}

A second experiment was conducted to further explore characteristics of duration discrimination. This experiment consisted of preliminary shaping of the temporal discrimination followed by four phases: Phase 1 involved shaping and development of a partial discrimination; Phase 2 involved extended training with fixed $T$ values to determine whether or not the performance approximated a stationary stochastic process; Phase 3 involved altering the reinforcement schedules (introducing a partial reinforcement schedule for some birds); and finally, Phase 4 involved several sessions of extinction training.

\section{Apparatus}

The apparatus was the same as that employed in Experiment 1 except that two chambers and their associated control equipment were employed.

\section{Subjects}

The Ss were 16 male White Carneaux pigeons, experimentally naive, ranging from 5 to 9 years of age at the start of the experiment, and varying in weight from 438 to $496 \mathrm{~g}$.

\section{Procedure}

The trial structure of this experiment was basically the same as in Experiment 1 except for a few minor modifications. The ITI varied randomly from trial to trial between the range of 10 to $20 \mathrm{sec}$ and was reset to $15 \mathrm{sec}$ whenever a response was made before it had elapsed. All food was obtained in daily experimental sessions during 5 days of the week, providing body weight was $80 \% \pm 10 \mathrm{~g}$ ad lib feeding weight. Ss were fed in their home cages during weekends. The shaping procedure employed in Experiment 1 was again utilized to develop the original temporal discrimination.

Phase 1. During this phase, the difference between $T_{1}$ and $T_{2}$ was gradually reduced to a level where the animals would maintain a partial discrimination with about $65 \%$ correct responses. The results of Experiment 1 indicated that this type of performance could be expected if $T_{1}$ was $4 \mathrm{sec}$ and $T_{2}$ was 5 sec. Accordingly, during Phase 1 of this experiment $T_{2}$ was fixed at $5 \mathrm{sec}$, while $T_{1}$ was successively increased from an initial value of $1 \mathrm{sec}$ to a final value of 4 sec. While each animal went through precisely the same sequence of $T_{t}$ values, the number of sessions it spent at each value depended on how quickly discrimination stabilized following each change. This was determined subjectively by visual inspection of the bird's performance. The sequence of $T_{1}$ values for all birds was as follows: $1,2,2.5,3$, $3.25,3.5$, and $4 \mathrm{sec}$. The number of sessions at each value for each animal beginning at $T_{1}$ equal to $2 \mathrm{sec}$ is indicated in Fig. 2. After $T_{1}$ had assumed its final value of $4 \mathrm{sec}$ for 10 to 12 sessions the reinforcement was decreased to $2-\mathrm{sec}$ access to grain to prevent the weight gain that had begun to occur with 90 daily 3 -sec reinforcements.

Phase 2. Sessions with $T_{1}$ equal to $4 \mathrm{sec}$ and with reinforcement equal to $2 \mathrm{sec}$ continued until behavior satisfied a criterion of stochastic stationarity. This was assessed for each bird by a chi-square test for stationarity over the last 12 sessions (Suppes \& Atkinson, 1960, p. 56). Under the null hypothesis, $P\left(R_{2} \mid T_{i}\right)$ for the first six sessions is assumed not to differ from $P\left(R_{2} \mid T_{i}\right)$ for the last six sessions; thus, the best predicted value of these probabilities is taken to be the mean $\mathbf{P}\left(\mathbf{R}_{2} \mid \mathrm{T}_{\mathrm{i}}\right)$ over all 12 sessions. A chi square with $p>.01$ was defined as indicating no difference and was considered to indicate stationarity between the first and last half. Since "stationarity" was behavior-dependent, the number of sessions each $S$ was given during this phase varied, ranging between 18 and 41 sessions.

Phase 3. In this phase, the probability of reinforcement was altered. It will be convenient to designate the probability of reinforcement for correct response $R_{i} \mid T_{i}$ as $\pi_{i}$, where $i$ can take two values, either 1 or 2 . The probability of reinforcement for an incorrect response was always zero.

The 16 birds were divided randomly into four groups of four birds each, with the following values of $\pi_{1}$ and $\pi_{2}$, respectively: Group 1, .7 and .7; Group 2, .7 and 1.0; Group 3, 1.0 and .7; and Group 4, 1.0 and 1.0. Binomial sequences in which $\pi$ was .7 determined the presentation schedule of reinforcement.

Note that the condition in Group 4 constituted no change from the prevailing condition in Phase 2. For Group 1, the probability of reinforcement was equally reduced for both types of correct response. For both these groups the reinforcement probability was symmetrical over the possible correct responses. For Groups 2 and 3 , however, $\pi_{i}$ was asymmetrically distributed over response outcomes.

The birds were given a minimum of 24 sessions; additional sessions were presented until response frequencies over the final 12 sessions satisfied the stationarity criterion used for Phase 2. The number of sessions required for stationarity varied from 24 to 44.

Phase 4. After achieving stationarity in Phase 3, Ss were given five 1-h extinction sessions on consecutive days.

\section{Results}

Figure 2 shows the estimates, $p_{1}$ and $p_{2}$, for single sessions throughout Experiment 2. Solid vertical lines demarcate the separate phases and dotted vertical lines indicate the point at which $T_{1}$ increased for each bird. All other statistics reported for Phases 2 and 3 were based on the final 12 sessions for each phase.

The major feature of the Phase 1 data in Fig. 2 is obvious by inspection: The accuracy of discrimination gradually diminished as $T_{1}$ was increased, with some evidence of improvement with practice following each change. Further comments on these data will be made later in the discussion section.

After the stationarity criterion had been met, with $T_{1}$ equal to $4 \mathrm{sec}$ and $T_{2}$ equal to $5 \mathrm{sec}$, the probability estimates, $\mathrm{p}_{1}, \mathrm{p}_{2}$, $p_{C}$, and $p_{L}$, were derived for each bird. $A$ general picture of the wide range in performance that was observed in this sample of birds can be obtained from Fig. 3 which shows $\hat{p}_{1}$ and $\hat{p}_{2}$ of each of the birds in ROC graphs. The open symbols in Figs. $3 \mathrm{~A}, 3 \mathrm{~B}, 3 \mathrm{C}$, and $3 \mathrm{D}$ represent Phase 2. The mean $\hat{p}_{C}$ over all birds was .659 and the mean $\hat{p}_{\mathrm{L}}$ was .519 ; however, in view of the clear individual differences, these means should be interpreted with caution.

A particular interest of the present experiment was to obtain detailed information of trial-by-trial changes in performance. One way of evaluating such fluctuations in performance as may occur between Trial $n$ and Trial $n-1$ is to derive estimates of the two probabilities: 

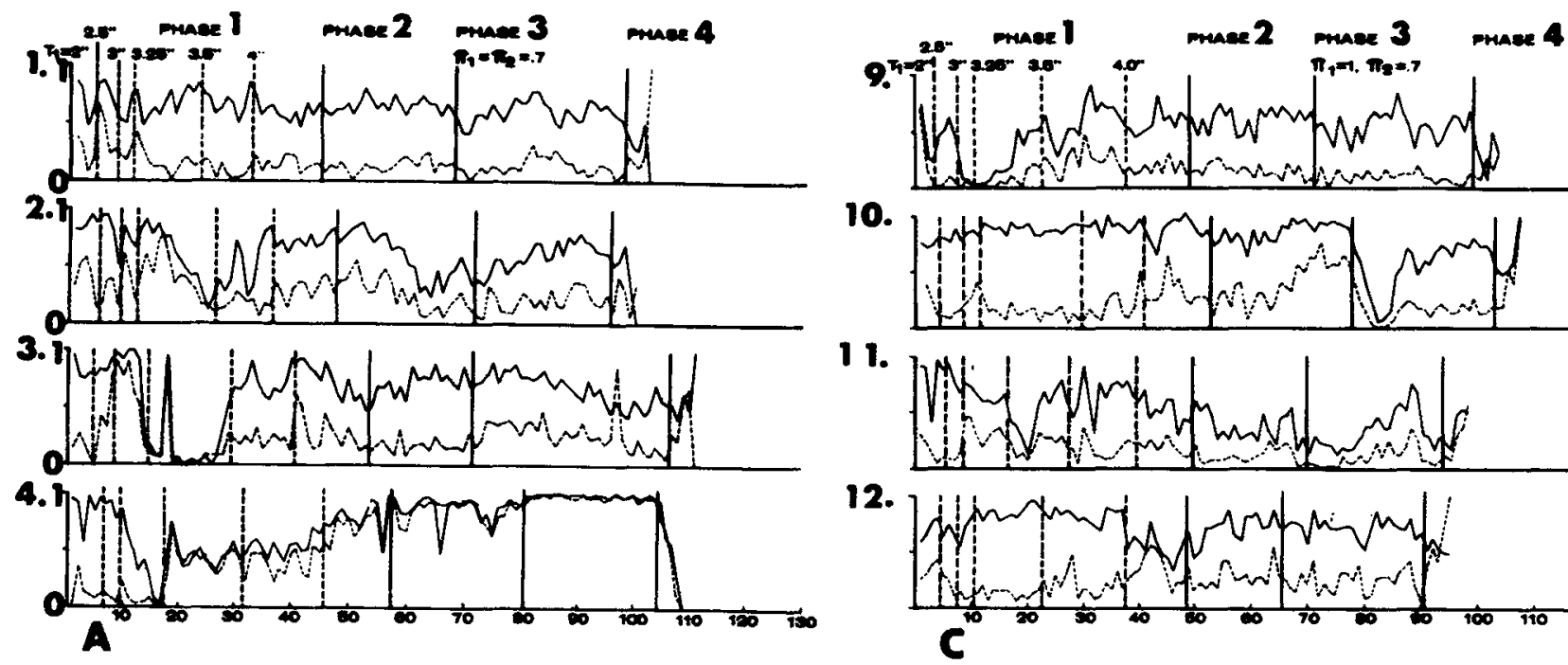

10.

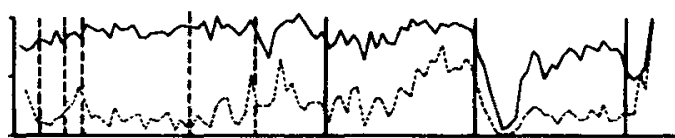

11.
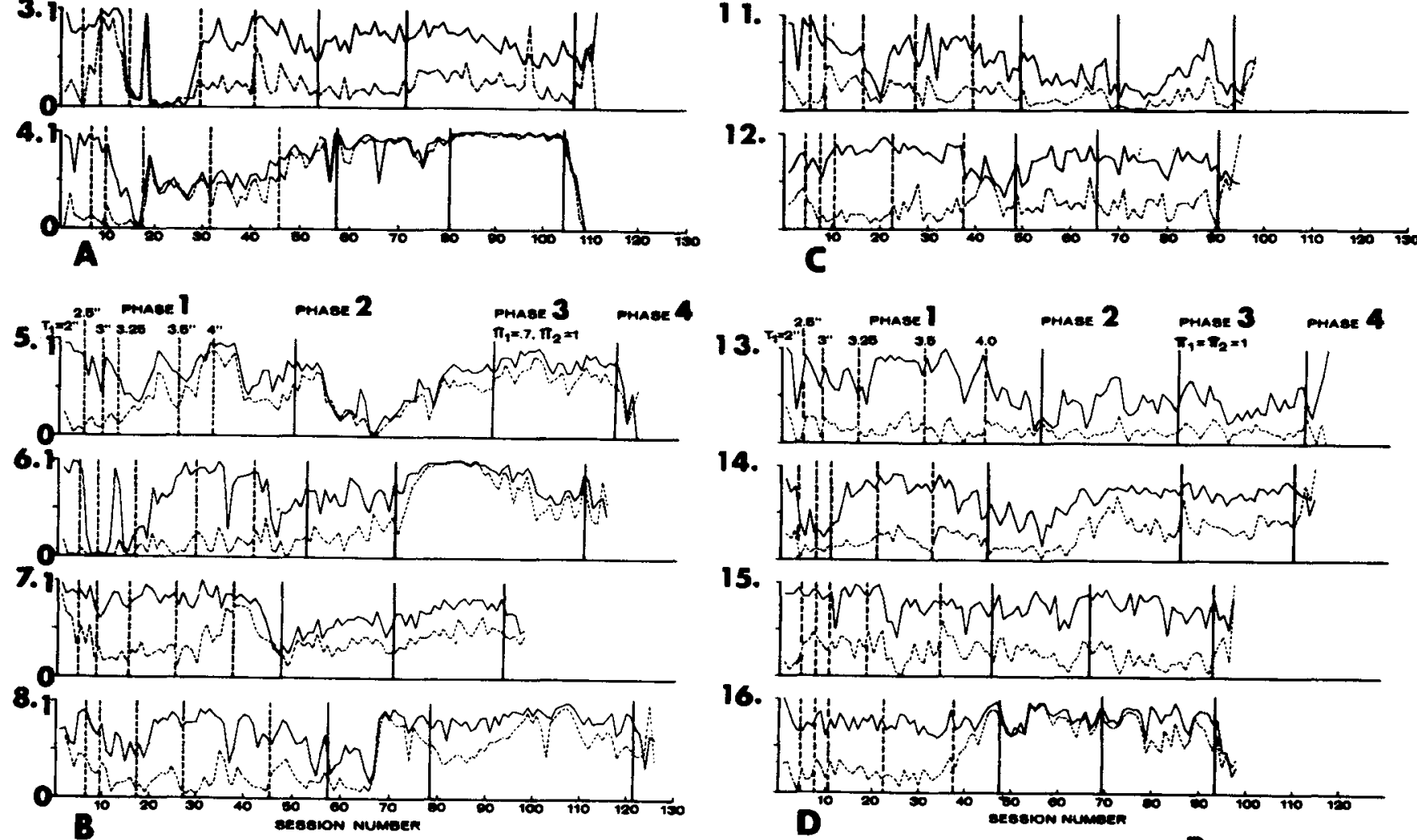

12.
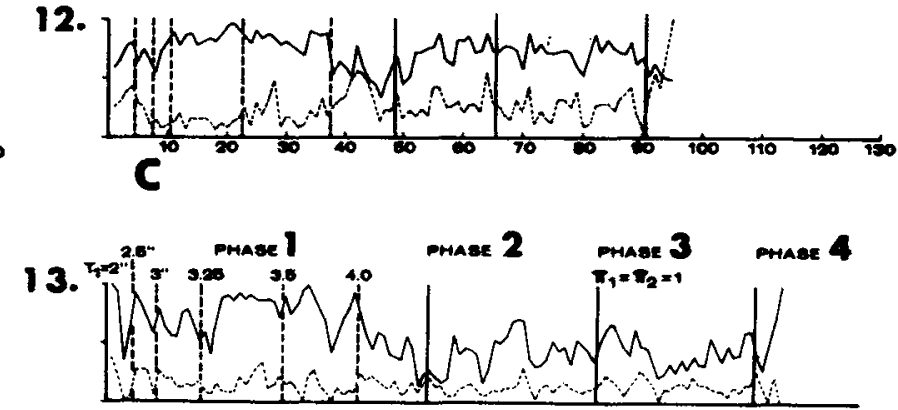

14.

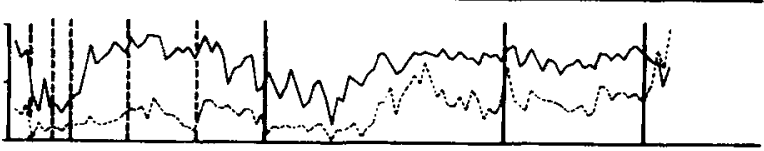

15.

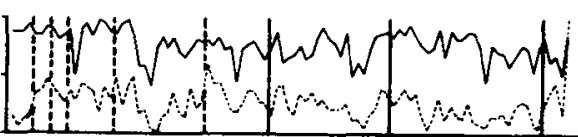

16.

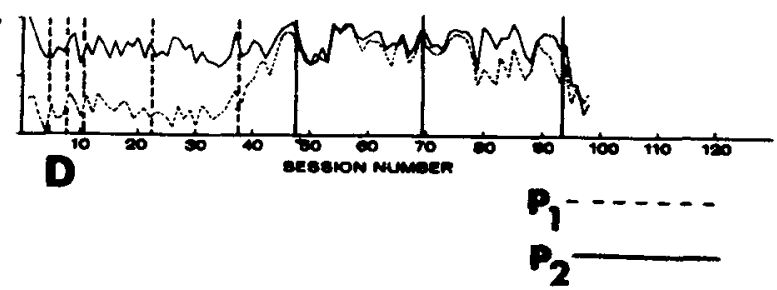

Fig. 2. Estimates of $p_{1}$ and $p_{2}$ for single sessions throughout Experiment 2. Individual bird numbers are shown beside each graph. $A$ is Group 1, B is Group 2, C is Group 3, and D is Group 4.

$P\left(R_{2, n} \mid T_{2, n} R_{j, n-1} T_{i, n-1} E_{k, n-1}\right)$ and $P\left(R_{2, n} \mid T_{1, n} R_{j, n-1} T_{i, n-1} E_{k, n-1}\right)$, where $R$ designates the response variable, $T$ the stimulus variable, and $E$ the reinforcement variable, for $i, j$ equal to 1 or 2 , and $\mathrm{k}$ equal to 1,2 , or 0 . There were four pairs of conditional $p_{2}$ and $p_{1}$ values. These first-order conditional probabilities are plotted in Fig. 4.

Figure 4A gives a general idea of how points are ordered in the total performance space. Figure 4B gives a much enlarged view of a subregion of the performance space to indicate how the performances were arrayed, although the reader should be careful to notice that the scale range is not the same for all graphs in Fig. 4B. In general the open triangle appears to be upper rightmost of the points in Fig. 4B.
No clear indication of other sequential dependencies emerges in consistent fashion for all birds. An order test (Suppes \& Atkinson, 1960) was carried out over all response frequencies for individual birds and was significant $(p<.01)$ for six of the birds, those marked with a star; the pooled chi square was also significant $(p<.01)$. The mean associative strength as indicated by the $\phi$ coefficient was .078 (Hays, 1963). Thus, there is an indication of a statistically significant, but very weak, relationship between the events on one trial and performance on the next trial.

It is of interest to consider to what extent the response tendency on Trial $n$ is conditioned on the stimulus of the preceding trial, which may be evaluated by suppressing the response and reinforcement outcomes on the previous trial. There are two possible stimulus events on Train $n-1, T_{1}$ and $T_{2}$. Therefore, there are two conditional proportions $P\left(R_{2, n} \mid T_{1, n-1}\right)$ and $P\left(R_{2, n} \mid T_{2, n-1}\right)$. Similar estimates may be derived that are conditional on the response event on Trial $n-1$, and on the reinforcing event on Trial $n-1$. These three types of conditional probability estimates are presented in Table 2. Table 2 also contains estimates of the probability of correct response on Trial $n$, following a correct and following an incorrect response on the preceding trial. These are denoted $\hat{\mathrm{P}}\left(\mathrm{C}_{1} \mid \mathrm{C}_{\mathrm{m}}\right)$, where $\mathrm{m}$ equals 1 or 0 . $\mathrm{M}$ equal to 1 represents a correct response, and $\mathrm{m}$ equal to 0 denotes an incorrect response. 

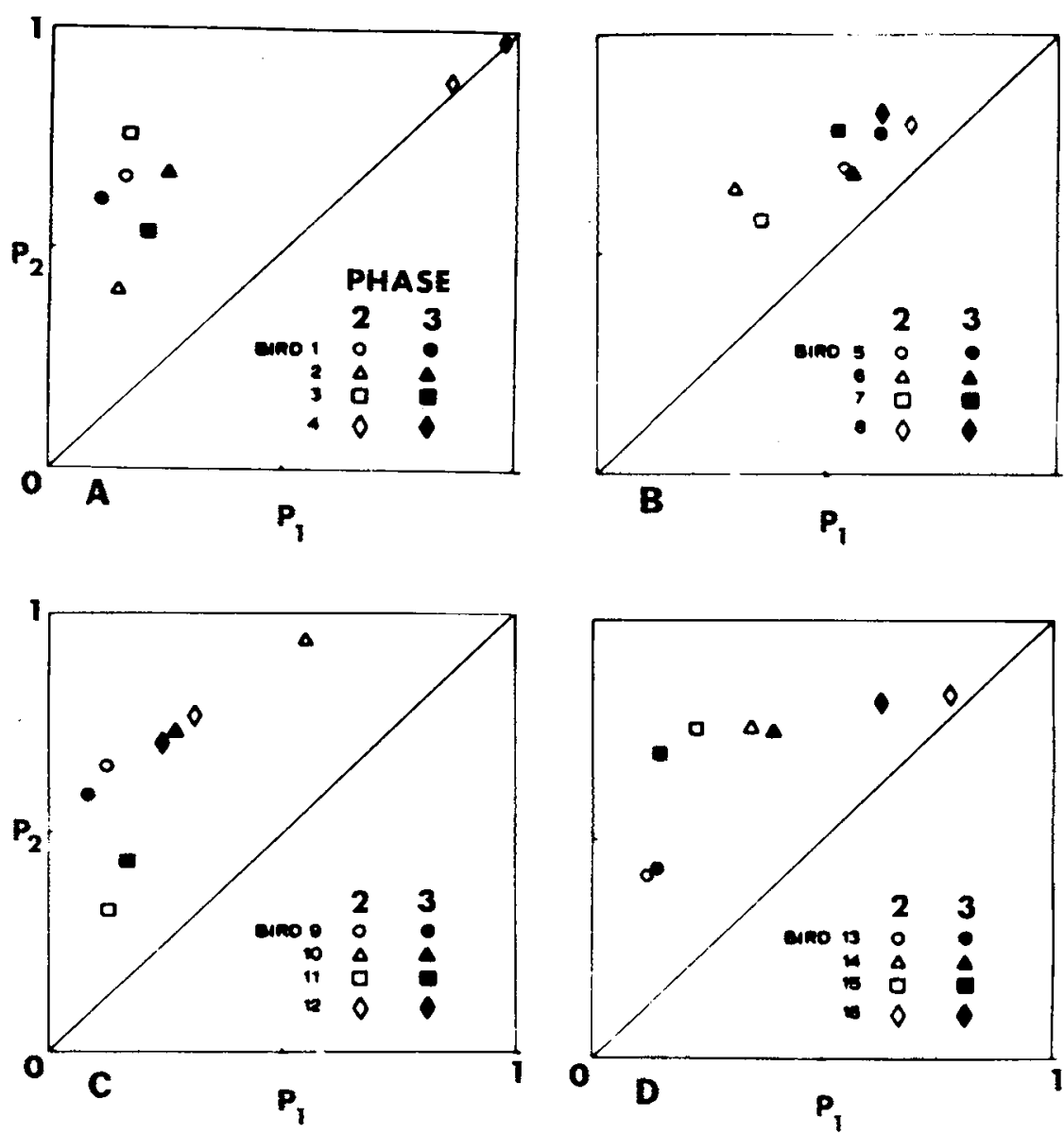

Fig. 3. ROC graphs showing estimates of $p_{1}$ and $p_{2}$ for individual birds in Phases 2 and 3 . A, Group 1; B, Group 2; C, Group 3; D, Group 4 .

For 13 of the 16 birds $\hat{\mathbf{P}}\left(\mathrm{R}_{2} \mid \mathrm{R}_{1}\right)<$ $\hat{\mathrm{P}}\left(\mathrm{R}_{2} \mid \mathrm{R}_{2}\right)$. In a sign test on the data, $\mathrm{p}$ equals .022 . Eleven of the 16 birds showed a tendency for $\hat{\mathrm{P}}\left(\mathrm{R}_{2} \mid \mathrm{E}_{1}\right)<\left(\mathrm{R}_{2} \mid \mathrm{E}_{2}\right)$. There appeared to be an effect of nonreinforcement on the preceding trial for some of the birds as well. However, the results were not significant in a sign test of the data. There did not appear to be first-order effects that were consistent across all birds in the other two types of conditional probabilities considered.

In addition to the events of the directly preceding trial, events over a longer series of previous trials might influence the performance in this type of discrimination
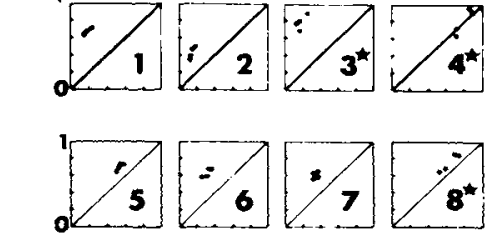

$\mathbf{P}_{\mathbf{2}}$,
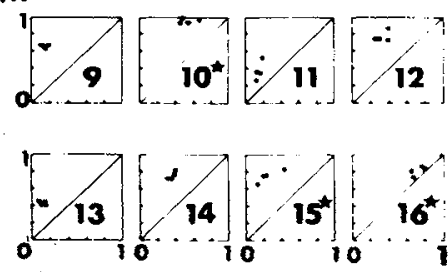

A
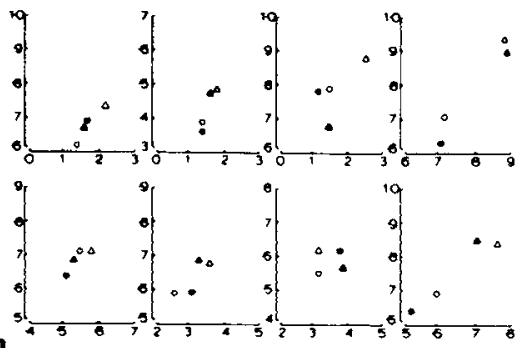

$\mathbf{P}_{\mathbf{2}^{\prime}} \mathbf{n}$

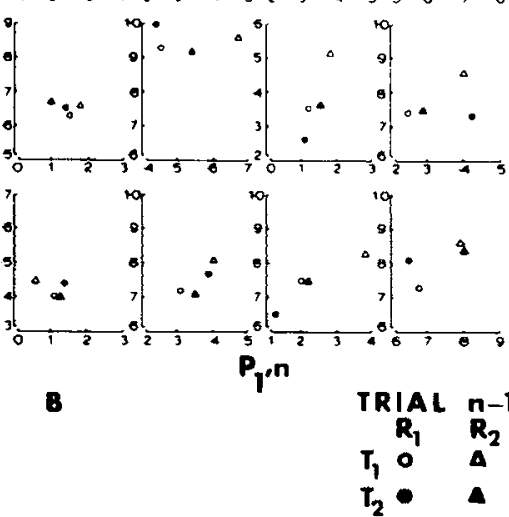

Fig. 4. First-order conditional probabilities for individual birds in Phase 2 . A shows the general position of the points in a separate ROC graph for each bird along with the $S$ number. B shows a more detailed view of a subregion of each graph.

Table 2

First-Order Conditional Probability Estimates

\begin{tabular}{|c|c|c|c|c|c|c|c|c|c|}
\hline Bird & $\dot{\mathrm{P}}\left(\mathrm{R}_{2} \mid \mathrm{T}_{2}\right)$ & $\hat{\mathbf{P}}\left(\mathrm{R}_{2} \mid \mathrm{T}_{1}\right)$ & $\hat{\mathbf{P}}\left(\mathbf{R}_{2} \mid \mathbf{R}_{2}\right)$ & $\hat{\mathbf{P}}\left(\mathbf{R}_{2} \mid \mathbf{R}_{1}\right)$ & $\hat{\mathbf{P}}\left(\mathrm{R}_{2} \mid \mathrm{E}_{1}\right)$ & $\hat{\mathrm{P}}\left(\mathrm{R}_{2} \mid \mathrm{E}_{2}\right)$ & $\hat{\mathbf{P}}\left(\mathbf{R}_{2} \mid \mathbf{E}_{0}\right)$ & $\hat{\mathrm{P}}\left(\mathrm{C} \mid \mathrm{C}_{1}\right)$ & $\hat{\mathbf{P}}\left(\mathrm{C} \mid \mathrm{C}_{0}\right)$ \\
\hline 1 & .413 & .395 & .427 & .388 & .381 & .417 & .425 & .747 & .763 \\
\hline 2 & .275 & .273 & .320 & .257 & .261 & .313 & .267 & .633 & .620 \\
\hline 3 & .431 & .486 & .451 & .465 & .469 & .525 & .498 & .790 & .823 \\
\hline 4 & .867 & .878 & .898 & .697 & .714 & .892 & .878 & .513 & .521 \\
\hline 5 & .594 & .641 & .626 & .604 & .636 & .609 & .616 & .585 & .563 \\
\hline 6 & .491 & .423 & .515 & .433 & .419 & .508 & .492 & .676 & .649 \\
\hline 7. & .487 & .446 & .470 & .463 & .438 & .476 & .483 & .602 & .636 \\
\hline 8 & .733 & .752 & .784 & .622 & .646 & .774 & .746 & .571 & .552 \\
\hline 9 & .383 & .394 & .388 & .388 & .389 & .381 & .396 & .761 & .750 \\
\hline 10 & .731 & .761 & .762 & .699 & .704 & .736 & .790 & .711 & .636 \\
\hline 11 & .207 & .250 & .276 & .213 & .237 & .248 & .211 & .609 & .587 \\
\hline 12 & .534 & .541 & .554 & .518 & .497 & .521 & .610 & .737 & .702 \\
\hline 13 & .272 & .258 & .252 & .269 & .257 & .250 & .284 & .646 & .662 \\
\hline 14 & .535 & .558 & .556 & .536 & .521 & .521 & .686 & .691 & .706 \\
\hline 15 & .458 & .507 & .519 & .449 & .471 & .484 & .502 & .773 & .737 \\
\hline 16 & .804 & .799 & .823 & .715 & .701 & .819 & .811 & .551 & .551 \\
\hline Overall $\overline{\mathrm{X}}$ & .513 & .524 & .539 & .482 & .484 & .523 & .538 & .662 & .654 \\
\hline
\end{tabular}




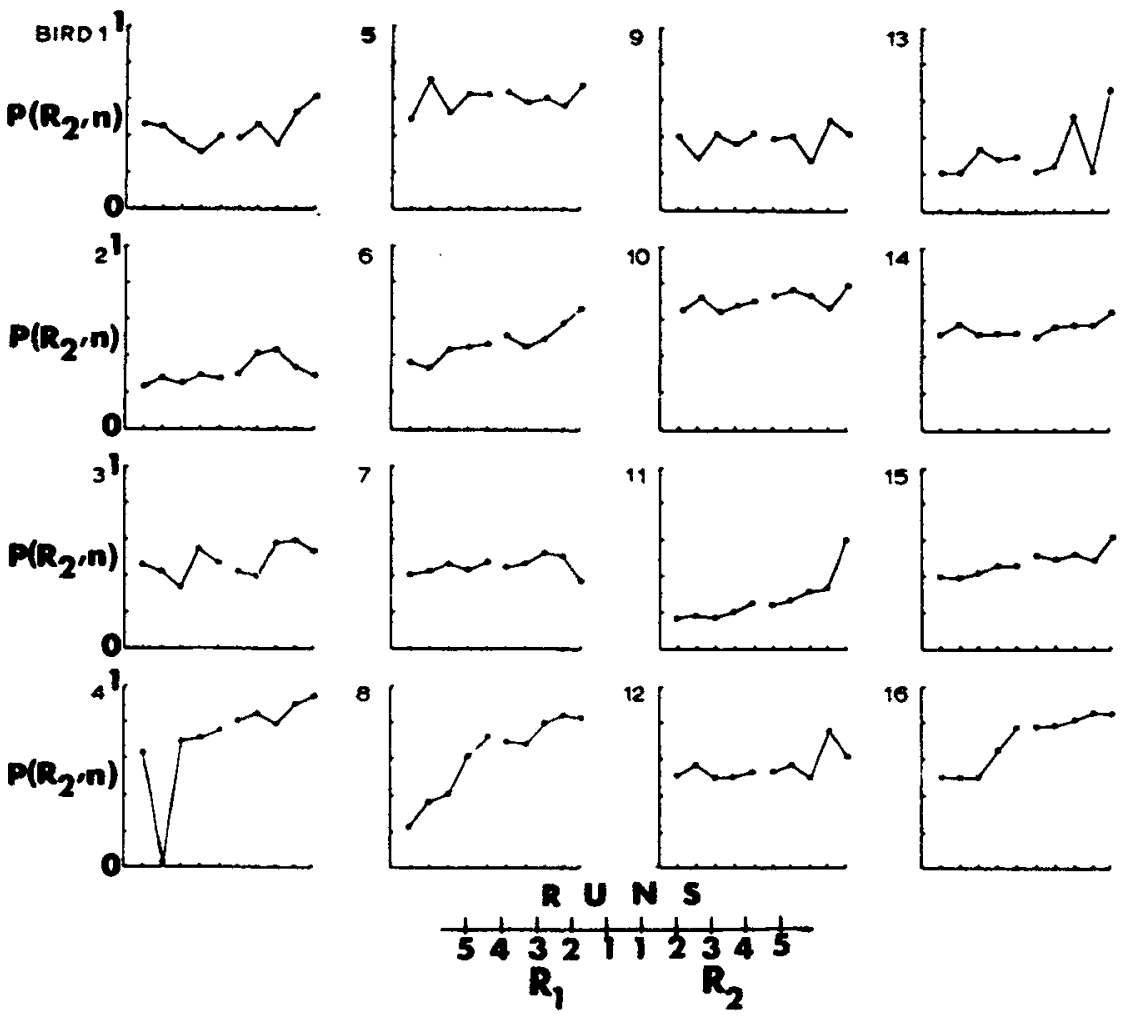

situation. There are a large number of ways to characterize events over a long series of trials. We shall only consider homogeneous runs of stimuli and responses on the $m$ trials preceding Trial $n$, where $m$ could equal $1,2,3,4$, or 5 . For obvious reasons these are referred to in the present discussion as "higher-order" conditional probabilities.

Higher-order probability estimates conditional on preceding response runs are shown graphically in Fig. 5.

For several birds, the plots have a positive slope, indicating the conditional $\mathbf{P}\left(\mathbf{R}_{2}\right)$ decreases with preceding runs of $\mathbf{R}_{1}$ and increases with preceding runs of $R_{2}$; cf. Birds 2, 4, 6, 8, 11, 13, 14, 15, and 16 . For no bird is a reverse slope indicated. Thus, there does appear to be a sequential effect conditional on response run length for some of the birds. Higher-order probability estimates conditional on preceding stimulus runs were also calculated; however, the sequential effects, if any, were small and were not systematic across all birds. ${ }^{4}$

The data from the final 12 sessions of Phase 3 were used to evaluate the effect that reduction of the probability of reinforcement had in this type of discrimination situation. The probabilities $\hat{\mathrm{p}}_{1}$ and $\hat{\mathrm{p}}_{2}$ are represented by the closed symbols in the four ROC graphs in Figs. $3 A, 3 B, 3 C$, and $3 D$, for Groups $1,2,3$, and 4 , respectively, and reflect the effect of altering this variable. The probabilities $\hat{\mathbf{p}}_{C}$ and $\hat{\mathrm{p}}_{\mathrm{L}}$ for Phases 2 and 3 are provided in Table 3.

For Group 1 birds, both $\pi_{1}$ and $\pi_{2}$ were decreased to .7. Apparently the decrement in probability of reinforcement altered performance of the birds in Group 1, but there was not a systematic effect, $P\left(E_{1} \mid R_{1} T_{1}\right)$, was reduced to .7 , and $\pi_{2}$ cf. Fig. $3 \mathrm{~A}$. For Group 2, $\pi_{1}$
Fig. 5. Higher-order conditional probabilities as a function of the preceding response run, for individual birds in Phase 2.

remained at 1.0. The effect of this, as can be seen in Fig. 3B, was to increase $p_{1}$ and $\mathrm{p}_{2}$ for all birds except Bird 8. Results for Group 3, in which $\pi_{2}, P\left(E_{2} \mid R_{2} T_{2}\right)$, was reduced to .7 while $\pi_{1}$ remained at 1.0 , are shown in Fig. 3C. The estimates for $p_{1}$ and $\mathrm{p}_{2}$ decreased for all birds except Bird 11 .

For Group 4 Fig. 3D, Phase 3 consisted of a minimum of 24 sessions after attainment of the stationarity criterion in Phase 2 during which there were no changes in the experimental contingencies. Consequently, Group 4 can be viewed as providing an indication of the way in which performance was modified as a result of extended exposure to this type of task. Birds 13 and 14 changed very little. Birds 15 and 16 appeared to develop slight changes in response tendency, but in opposite direction.

The mean $\mathrm{P}_{\mathrm{C}}$ over all birds, .663, did not represent an increase beyond $1 \sigma$ of its value in Phase 2, i.e., .659; and changes in $\mathrm{PC}_{\mathrm{C}}$ for individual birds were not systematic. When $p_{L}$ is compared for Phases 2 and 3 , it appears that the probability of an $R_{2}$ increased for Group 2 birds, with the exception of Bird 8, was decreased for Group 3 birds, with the exception of Bird 11, and was not systematically altered for the other two groups.

Finally, behavior of the birds during extinction, i.e., $\pi_{i}$ was reduced to 0 for $i$ equal to 1,2 , was evaluated over five consecutive daily sessions for $1 \mathrm{~h}$ each. Table 3

Estimates of $P_{C}$ and $P_{L}$

\begin{tabular}{|c|c|c|c|c|c|}
\hline \multirow[b]{2}{*}{ Group } & \multirow[b]{2}{*}{ Bird } & \multicolumn{2}{|c|}{$P_{C}$} & \multicolumn{2}{|c|}{$\mathbf{P}_{\mathbf{L}}$} \\
\hline & & Phase 2 & Phase 3 & Phase 2 & Phase 3 \\
\hline \multirow[t]{2}{*}{1} & $\begin{array}{l}1 \\
2 \\
3 \\
4\end{array}$ & $\begin{array}{l}.750 \\
.627 \\
.795 \\
.519\end{array}$ & $\begin{array}{l}.747 \\
.712 \\
.662 \\
.502\end{array}$ & $\begin{array}{l}.411 \\
.277 \\
.464 \\
.868\end{array}$ & $\begin{array}{l}.360 \\
.456 \\
.377 \\
.981\end{array}$ \\
\hline & $\overline{\mathrm{X}}$ & .693 & .656 & .505 & .544 \\
\hline 2 & $\begin{array}{r}5 \\
6 \\
7 \\
8 \\
\overline{\mathbf{x}}\end{array}$ & $\begin{array}{l}.577 \\
.672 \\
.614 \\
.564 \\
607\end{array}$ & $\begin{array}{l}.579 \\
.574 \\
.636 \\
.602 \\
508\end{array}$ & $\begin{array}{r}.619 \\
.474 \\
.466 \\
.740 \\
575\end{array}$ & $\begin{array}{l}.696 \\
.621 \\
.657 \\
.720 \\
674\end{array}$ \\
\hline \multirow[t]{2}{*}{3} & $\begin{array}{r}\boldsymbol{X} \\
9 \\
10 \\
11 \\
12\end{array}$ & $\begin{array}{l}.607 \\
.758 \\
.689 \\
.605 \\
.729\end{array}$ & $\begin{array}{l}.598 \\
.727 \\
.775 \\
.641 \\
.733\end{array}$ & $\begin{array}{l}.575 \\
.387 \\
.740 \\
.237 \\
.535\end{array}$ & $\begin{array}{l}.674 \\
.312 \\
.438 \\
.302 \\
.480\end{array}$ \\
\hline & $\overline{\mathbf{X}}$ & .695 & .719 & .475 & .383 \\
\hline \multirow[t]{2}{*}{4} & $\begin{array}{l}13 \\
14 \\
15 \\
16\end{array}$ & $\begin{array}{l}.652 \\
.701 \\
.764 \\
.531\end{array}$ & $\begin{array}{l}.652 \\
.678 \\
.775 \\
.607\end{array}$ & $\begin{array}{l}.266 \\
.542 \\
.486 \\
.794\end{array}$ & $\begin{array}{l}.283 \\
.564 \\
.412 \\
.724\end{array}$ \\
\hline & $\overline{\mathbf{X}}$ & .662 & .678 & .522 & .496 \\
\hline Overall & $\overline{\mathrm{X}}$ & .659 & .663 & .519 & .524 \\
\hline
\end{tabular}


Analysis of extinction results showed the mean number of trials for Groups 1, 2, 3, and 4 were $340,444,333$, and 337 , respectively. Variability among birds within groups was high, and there was considerable overlap between groups in number of trials. For most birds the number of trials dropped off rapidly after the first day of extinction; however, for Birds 2, 3, 5, 6, and 7 there was not a noticeable reduction in number of trials until Day 3 of extinction.

ROC graphs of $\hat{p}_{1}$ and $\hat{p}_{2}$ for successive days of extinction were examined; however, a group difference did not emerge, and neither did there appear to be a systematic trend among the birds. In general, no systematic effects, other than a decrease in the number of trials, occurred during extinction.

Response times during Phases 2 and 3 were recorded and analyzed; no large effects were observed. Analysis of the way in which performance might change over successive quartiles of a session was also carried out but no apparent trends were revealed.

\section{DISCUSSION}

In Fig. 3 it appeared that for most birds the increments in $T_{1}$ during Phase 1 occurred gradually enough so that the discrimination was not seriously disturbed until the final increment to $4 \mathrm{sec}$, which, for many birds, decreased $\hat{\mathrm{p}}_{2}$ and increased $\dot{\mathrm{p}}_{1}$. Where discrimination was weakened by an increment in $T_{1}$ there was not always an immediately discernable effect, cf. Birds 7, 11,13 , and 16 at the 4-sec increment.

Occasionally an apparent response fixation developed during Phase 1 , cf. 3 and 6 , but this was followed by improvement of the discrimination. Thus the presence of a strong fixation did not necessarily preclude subsequent development of good discriminative behavior. Fixations developed early in Phase 1 seemed typically to be toward $R_{1}$, the short stimulus response.

It will be recalled that after the $4-\mathrm{sec}$ increment in $T_{1}$ had been in effect for several sessions, reinforcement duration was reduced from 3 to $2 \mathrm{sec}$ for the remainder of the experiment. This decrease in the amount of reinforcement might have been expected to alter the performance in some fashion. With the exception, perhaps, of Bird 5, there is no strong evidence that this happened.

The only clear sequential effect found in the results of this experiment was a tendency on the part of some birds to repeat responses. This is consistent with studies of a psychophysical nature with humans in which response dependencies of this type have been observed (Verplanck,
Collier, \& Cotton, 1952; Collier \& Verplanck, 1958; Kinchla, 1966).

On the whole, the data are surprisingly similar to an independent-trials process and are not consistent with those detection models that predict specific sequential effects. For example, a variable-sensitivity model, in which both bias and sensitivity may vary from trial to trial (Atkinson, 1963), can be rejected, since in the present experiment there was no evidence that $\hat{\mathrm{P}}\left(\mathrm{C}_{1, n} \mid \mathrm{C}_{1, \mathrm{n}-1}\right)$ and $\hat{\mathrm{P}}\left(\mathrm{C}_{1, n} \mid \mathrm{C}_{0, n-1}\right)$ were unequal. The variable sensitivity model predicts a sequential dependency between $\mathrm{p}_{C}$ and the correctness of the previous trial. Note that a sequential effect of this sort has considerable intuitive appeal since it could be argued that an animal's efficiency or accuracy might slowly vary during a session; if so, his accuracy on one trial would be related to his accuracy on the subsequent trial. However, no empirical support for this argument was obtained.

Stimulus-dependent sequential properties have been found in human performance in psychophysical tasks by a number of investigators. However, strong sequential stimulus dependencies were not found in the present analysis, either in terms of bias or sensitivity shifts, so that models that predict such effects would seem inappropriate for this discrimination situation.

Learning models that predict bias shifts dependent on prior reinforcement are difficult to compare with the present situation, since they deal with nonresponse-contingent reinforcement events, and the present experiment used response-contingent reinforcement. This latter fact is relevant in evaluating the observed response dependencies. In Phase 2, the probability of reinforcement, given a correct response, was always equal to one, thus it is difficult to separate the effect of a response on $n-1$ from a reinforcing event on $n-1$ for this phase. In Phase 3, however, the effect of a reinforcement could be evaluated separately from the effect of a response on the preceding trial, since $P\left(E_{\mathfrak{i}} \mid T_{\mathfrak{i}} \mathbf{R}_{\mathbf{i}}\right)<1$, for $i$ equal to 1 (Group 2), 2 (Group 3), or both (Group 1). Trials following reinforced correct trials did not appear to differ from trials following unreinforced correct trials. Thus the response-response correlations observed in Phase 2 do not appear to be simply a reinforcement effect.

This result is particulary interesting in regard to the role of individual reinforcements. They did not seem to alter the probability of response in the way some learning models would suggest, e.g., a type of stimulus sampling process in which each reinforcement increases the probability of a particular response.

Stereotyped overt activity has been noted by many investigators in studies requiring the discrimination of temporal intervals. Similar types of overt activity were observed in this discrimination situation during the period that the tone was on. Visual monitoring of the birds revealed that for some birds this activity could be differentiated into $T_{1}$ and $T_{2}$ segments but not for all birds. Differentiation of the behavior while the tone was on did not appear to be correlated with the accuracy of discrimination.

The graphs of $\dot{\mathrm{p}}_{1}$ and $\hat{\mathrm{p}}_{2}$ in Fig. 2 provide an interesting view of how the assymetrical reduction in reinforcement manifested itself from session to session. Initial effects of the reduction can be seen to be different for individual cases. For instance, within a few sessions after the asymmetrical reduction in reinforcement Bird 6, Group 2, and Bird 10 in Group 3 adopted very strong fixations toward the response that had the higher reinforcement. probability. These were later modified and discrimination improved, although the response tendencies never returned to the earlier value, whereas for Bird 7 the alteration occurred gradually with $\hat{p}_{1}$ and $\hat{\mathrm{p}}_{2}$ shifting slowly upwards over a number of sessions. Thus it is difficult to characterize in a general fashion the way in which behavior changed prior to asymptotic performance.

A significant difference between Phase 2 and Phase 3 was found for all birds except 13 and 14. According to TSD, changing the "pay-off" structure should affect the response criterion but not the sensitivity. For Group 2, the criterion should shift to a lower value, increasing $\hat{\mathrm{p}}_{1}$ and $\hat{\mathrm{p}}_{2}$; for Group 3 the criterion should shift to a higher value, and $\hat{\mathrm{p}}_{1}$ and $\hat{\mathrm{p}}_{2}$ should decrease. Decreasing the payoff equally for both stimuli as in Group 1 is not predicted to have any effect on criterion or sensitivity.

The change in performance is in the predicted direction for Groups 2 and 3, with the exception of one bird in each group, 8 and 11. A relevant factor in considering the absence of an effect for these birds may be that each of these birds had adapted to a criterion in the direction of the predicted change before $\pi_{i}$ was reduced. The data do not allow satisfactory tests to be made for linearity of the functions since there are only two points per curve.

The systematic shifts shown by Groups 2 and 3 may be compared with the data for Groups 1 and 4 . Alterations in performance are not predicted by TSD for Group 1, and, while there were indications 
of shifts in criterion as well as in sensitivity, there did not appear to be uniform changes for all birds. Similar statements can be made for Group 4.

The theoretical measure, $\mathrm{d}^{\prime}$, was derived for these data. The mean value of $\mathrm{d}^{\prime}$ over all birds was .923 in Phase 2, and .934 in Phase 3. Shifts in $d^{\prime}$ did not appear correlated with reinforcement probability. Mean values of $d^{\prime}$ in Phases 2 and 3 . respectively, for Group 1 were .995 and .85; for Group 2 were .57 and .55 ; for Group 3, were 1.19 and 1.29; for Group 4 were .935 and 1.02. That is, in Groups 1 and 2, $\mathrm{d}^{\prime}$ was decremented, for Groups 3 and 4 it incremented. From these results it would appear that discriminability was not appreciably altered by the change to a partial reinforcement schedule in Phase 3.

In some situations, partial reinforcement has been found to produce greater resistance to extinction than continuous reinforcement (Kimble, 1961, Chap. 10). Extrapolation of this effect to the present situation might lead to the prediction that the number of trials in extinction would be ordered as follows: Group $1>$ Group 4, with Groups 2 and 3 at intermediate levels. The data did not conform to this prediction. Total mean number of trials for Group 1 was 340 and for Group 4 was 337. A Mann-Whitney $U$ test between the two groups was not significant.

Group 2 birds had the highest mean number of trials over the 5 days of extinction, and for three of the four birds in Group 2 there was a slower decrement in number of daily trials during the course of extinction. However, considerable overlap between groups was noted. In conclusion, the performance during extinction did not appear to be related to the partial reinforcement schedules that obtained in Phase 3 in any clear way.
Other investigators (Blough, 1966; Jenkins \& Harrison, 1960) have found evidence that discrimination improved in extinction, as revealed by a sharpening of stimulus generalization curves. However, no general trend toward improved discrimination in extinction was found in this experiment.

\section{REFERENCES}

ATKINSON, R. C. A variable sensitivity theory of signal detection. Psychological Review, $1963,70,91-106$

BL.OUGH, D. S. The response character of stimulus control functions. Paper read at Eastern Psychological Association, New York, April 1966.

COLLIER, w. G., \& VERPLANCK, w. S. Non independence of successive responses at the visual threshold as a (f) interpolated stimuli. Journal of Experimental Psychology, 1958, $55,429-437$.

CREELMAN, C. D. Human discrimination of auditory duration. Journal of the Acoustical Society of America, 1962, 34, 582-593.

GREEN, D. M., \& SWETS, J. A. Signal detection theory and psychophysics New York: Wiley, 1966.

HACK, M. H. Signal detection in the rat. Science, $1963,00,758-759$.

HAYS, W. L. Statistics for psychologists New York: Holt, Rinehart \& Winston, 1963.

JENKINS, H. M., \& HARRISON, R. H. Effect of discrimination training on auditory generalization. Joumal of Experimental Psychology, 1960, 59, 246-253.

KEUCHLER, H. A. Signal detectability and the response criterion in the pigeon. Paper presented at Eastern Psychological Association, Washington, D.C., April 1968.

KIMBLE, G. A. Hilgard and Marquis' Conditioning and leaming. New York: Appleton-Century-Crofts, 1961.

KINCHLA, R. A. A comparison of sequential effects in detection and recognition. Experimental Psychology Series, New York University. Technical Report No. 1, 1966.

MORRISON, G. R. Models for visual detection in the rat. Psychology Series, McMaster University, Technical Report No. 8, 1967.

MORRISON, G. R., \& NORRISON, W. Taste and detection in the rat. Canadian Joumal of
Psychology, 1966, 20, 208-217.

NEVIN, J. A. A method for the determination of psychophysical functions in the rat. Journal of Experimental Analysis of Behavior, 1964, 7, 169.

STUBBS, A. The discrimination of stimulus duration by pigeons. Joumal of Experimental Analy sis of Behavior, 1968, 11, 223-238.

SUPPES, P., \& ATKINSON, R. C. Markov learning models for multi-person interactions. Stanford: Stanford University Press, 1960. (P. 56-test for stationarity; p. 57-an order test.)

TERRACE, H. S. Stimulus control. In W. K. Honig (Ed.), Operant behavior: Areas of research and application. New York: Appleton-Century-Crofts, 1966. Pp. 271-344.

VERPLANCK, W. S., COLLIER, G. H., \& COTTON, J. W. Non independence of successive responses in measures of the visual threshold. Journal of Experimental Psychology, 1952, 44, 273-282.

\section{NOTES}

1. This article is based on a dissertation submitted to the Department of Psychology, McMaster University, Hamilton, Ontario, in partial fulfillment of the requirements for the $\mathrm{PhD}$ degree. The author would like to express her appreciation to Drs. R. A. Kinchla, H.M. Jenkins, and G. R. Morrison for their assistance, and also to Larry Arnold for helping run Experiment 2.

2. Address: Auditory Research Laboratory, Psychology Department, Princeton University, Princeton, N.J.

3. Originally all eight birds were thought to be male, but subsequent to the experiment one bird, No. 3, was found to be female. Only the results for the seven male birds are discussed.

4. A more detailed account of the sequential analysis may be found in the author's PhD dissertation, "Discrimination of two auditory durations by pigeons," McMaster University, 1969.

(Accepted for publication January 2, 1970.) 